\title{
Learning from demonstration and adaptation of biped locomotion
}

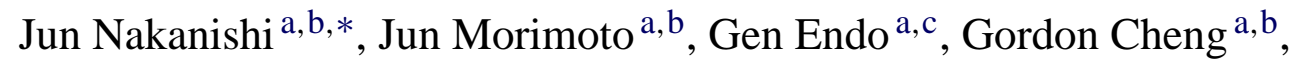 \\ Stefan Schaal ${ }^{\mathrm{a}, \mathrm{d}}$, Mitsuo Kawato ${ }^{\mathrm{a}, \mathrm{b}}$ \\ a ATR Computational Neuroscience Laboratories, 2-2 Hikaridai, Seika-cho, Soraku-gun, Kyoto 619-0288, Japan \\ ${ }^{\mathrm{b}}$ ICORP, Japan Science and Technology Agency, 2-2 Hikaridai, Seika-cho, Soraku-gun, Kyoto 619-0288, Japan \\ ${ }^{c}$ Sony Corporation, 6-7-35 Kitashinagawa, Shinagawa-ku, Tokyo 141-0001, Japan \\ d Department of Computer Science, University of Southern California, 3641 Watt way, Los Angeles, CA 90089-2520, USA
}

\begin{abstract}
In this paper, we introduce a framework for learning biped locomotion using dynamical movement primitives based on non-linear oscillators. Our ultimate goal is to establish a design principle of a controller in order to achieve natural human-like locomotion. We suggest dynamical movement primitives as a central pattern generator (CPG) of a biped robot, an approach we have previously proposed for learning and encoding complex human movements. Demonstrated trajectories are learned through movement primitives by locally weighted regression, and the frequency of the learned trajectories is adjusted automatically by a novel frequency adaptation algorithm based on phase resetting and entrainment of coupled oscillators. Numerical simulations and experimental implementation on a physical robot demonstrate the effectiveness of the proposed locomotion controller. (C) 2004 Elsevier B.V. All rights reserved.
\end{abstract}

Keywords: Biped locomotion; Learning from demonstration; Dynamical movement primitives; Phase resetting; Frequency adaptation

\section{Introduction}

There has been a growing interest in biped locomotion with the recent development of advanced humanoid robots. Many of existing successful walking algorithms use the zero moment point (ZMP) criterion [1] for off-line motion planning [2,3] and on-line balance compensation [4-6]. These ZMP-based methods have been shown to be effective to achieve biped locomotion in legged robots with flat feet. However, they require precise modeling of the robot dynamics and high-gain trajectory tracking control, and the generated patterns result in a typical 'bent-knee' posture to avoid singularities of inverse kinematics. From the

\footnotetext{
* Corresponding author.

E-mail address: jun@atr.jp (J. Nakanishi).
}

viewpoint of energy efficiency, such walking patterns are not desirable since torque must be continuously applied to the knee joint to maintain a bent-knee posture. These previous ZMP approaches have primarily focused on the ability of executing planned movements at any instance by ensuring surface contact between the sole and the ground [7] rather than natural human-like motion which exploits passive dynamics of the body [8].

In contrast to off-line trajectory planning, biologically inspired control approaches based on central pattern generators (CPGs) with neural oscillators have been drawing much attention for rhythmic motion generation. As a CPG, a neural oscillator proposed by Matsuoka [9] is widely used, which models the firing rate of two mutually inhibiting neurons described in a set of differential equations. This model is used 
in robotic applications to achieve designated tasks involving rhythmic motion which requires interactions between the system and the environment. Examples include biped locomotion [10-13], quadruped locomotion [14], juggling [15], drumming [16], and playing with a slinky toy [17]. Neural oscillators have desirable properties such as adaptation to the environment through entrainment. However, it is difficult to design interconnection and feedback pathways of neural oscillators, and to manually tune all open parameters in order to achieve the desired behavior.

Other approaches include [18] in an effort to design a simple controller based on physical intuition. The control strategy proposed in Ref. [18] is quite simple and easy to implement. However, it requires manual tuning of the control parameters and accurate torque-controlled actuators.

In this paper, we suggest an approach to learning biped locomotion from demonstration and its adaptation through coupling between the pattern generator and the mechanical system. Motivated by human's capability of learning and imitating demonstrated movements of a teacher, imitation learning has been explored as an efficient method for motor learning in robots to accomplish desired movements [19-21]. In our previous work, we proposed dynamical movement primitives to encode complex discrete and rhythmic multi-joint movements through imitation learning [22]. Dynamical movement primitives are formulated as a set of autonomous non-linear differential equations with well-defined attractor dynamics. Demonstrated trajectories are learned using locally weighted regression, and the output of dynamical movement primitives serves as kinematic movement plans, e.g., desired trajectories, for a robot.

This paper presents the idea of using the rhythmic movement primitives based on phase oscillators [22] as a CPG to learn biped locomotion from demonstration. Compared with neural oscillators, one of the appealing properties of phase oscillators is that the desired phase relationship among oscillators can be specified in a straightforward manner. In Ref. [23], a comprehensive formulation of phase coordination of coupled phase oscillators is proposed. Applications of coupled phase oscillators have been explored in the gait control of multi-legged robots [24,25] and the control of a biped robot [26]. In addition to using phase oscillators, our movement primitive has various desirable properties which are beneficial for biped locomotion. For example, it can learn a demonstrated trajectory rapidly, and it is easy to re-scale the learned rhythmic movement in terms of amplitude, frequency and offset of the patterns [22]. In the application of rhythmic movement primitives to biped locomotion, we introduce coupling terms to the movement primitives to achieve the desired phase relationship among limbs following the formulation proposed in Ref. [23]. We also propose an adaptation algorithm for the frequency of walking based on phase resetting [27] and entrainment between the phase oscillator and mechanical system using feedback from the environment. Frequency adaptation of a CPG is beneficial when the desired frequency of the coupled system is not exactly known in advance.

In Ref. [26], a similar idea of using coupled phase oscillators as a pattern generator for biped locomotion was proposed. In their method, desired joint trajectories of the legs are generated from a nominal trajectory at the tip of each leg defined by a combination of simple prescribed functions of phase through inverse kinematics [26]. In comparison to Ref. [26], we believe that our method has the advantage of flexibility in encoding complex movements by imitation learning and the potential capability of improving learned movements through reinforcement learning [28]. We demonstrate the effectiveness of the proposed control strategy by numerical simulations and experimental implementation.

\section{Biped robot}

We use a planar 5-link biped robot developed in Ref. [29]. The height of the robot is $0.4 \mathrm{~m}$ and the weight is about $2 \mathrm{~kg}$. The length of each link of the leg is $0.2 \mathrm{~m}$. The mass of the body is $1.0 \mathrm{~kg}$, the thigh is $0.43 \mathrm{~kg}$ and the shank is $0.05 \mathrm{~kg}$. The motion of the robot is constrained within the sagittal plane by a tether boom. The hip joints are directly actuated by direct drive motors, and the knee joints are driven by direct drive motors through a wire transmission mechanism with the reduction ratio of 2.0. These transmission mechanisms with low reduction ratio provide high back drivability at the joints. Foot contact with the ground is detected by foot switches. The robot is an underactuated system having rounded soles with no ankles. Thus, it 
is challenging to design a controller to achieve biped locomotion with this robot since no actuation can be applied between the stance leg and the ground compared to many of the existing biped robots which have flat feet with ankle joint actuation.

\section{Dynamical movement primitives}

In this section, we outline the rhythmic dynamical movement primitives originally proposed in Ref. [22], which we will use as a CPG for biped locomotion in this paper.

\subsection{Rhythmic dynamical movement primitives}

Rhythmic dynamical movement primitives encode periodic behavioral patterns as an output of a set of non-linear dynamical systems composed of a canonical dynamical system with a phase oscillator and a transformation dynamical system with a non-linear function approximator.

Consider the following limit cycle oscillator characterized in terms of an amplitude $r$ and a phase $\phi$ as a canonical dynamical system which generates basic rhythmic patterns:

$\tau \dot{\phi}=1$,

$\tau \dot{r}=-\mu\left(r-r_{0}\right)$,

where $\tau$ is a temporal scaling factor, $r_{0}$ determines the desired (relative) amplitude, and $\mu$ is a positive constant. Note that the phase dynamics (1) can be written as

$\dot{\phi}=\omega$,

where $\omega=1 / \tau$ is the natural frequency. When there are multiple oscillators, we will introduce coupling terms among the oscillators (see Section 4.1). This rhythmic canonical system is designed to provide an amplitude signal $\tilde{\mathbf{v}}=[r \cos \phi, r \sin \phi]^{T}$ and phase variable $\bmod (\phi, 2 \pi)$ to the following second-order transformation dynamical system $(z, y)$, where the output $y$ is used as the desired trajectory for the robot:

$\tau \dot{z}=\alpha_{z}\left(\beta_{z}\left(y_{m}-y\right)-z\right)$,

$\tau \dot{y}=z+f(\tilde{\mathbf{v}}, \phi)$, where $\alpha$ and $\beta$ are time constants, $y_{m}$ is an offset of the output trajectory. $f$ is a non-linear function approximator using local linear models [30] of the form

$f(\tilde{\mathbf{v}}, \phi)=\frac{\sum_{k=1}^{N} \Psi_{k} \mathbf{w}_{k}^{T} \tilde{\mathbf{v}}}{\sum_{k=1}^{N} \Psi_{k}}$,

where $\mathbf{w}_{k}$ is the parameter vector of the $k$ th local model which will be determined by locally weighted learning [30] from a demonstrated trajectory $y_{\text {demo }}$ (see Section 3.2). Each local model is weighted by a Gaussian kernel function

$\Psi_{k}=\exp \left(-h_{k}\left(\bmod (\phi, 2 \pi)-c_{k}\right)^{2}\right)$,

where $c_{k}$ is the center of the $k$ th linear model, and $h_{k}$ characterizes its width. A final prediction is calculated by the weighted average of the predictions of the individual models. As demonstrated in Ref. [22], the amplitude, frequency and offset of the learned rhythmic patterns can be easily modified by scaling the parameters $r_{0}, \omega(=1 / \tau)$ and $y_{m}$ individually.

\subsection{Imitation learning with dynamical movement primitives}

An important issue is how to learn the parameters $\mathbf{w}_{k}$ in the non-linear function (6) to characterize the output of a dynamical movement primitive for a given demonstrated trajectory $y_{\text {demo }}$. Given a sampled data point $\left(f_{\text {target }}, \tilde{\mathbf{v}}\right)$ at $t$ where

$f_{\text {target }}=\tau \dot{y}_{\text {demo }}-z_{\text {demo }}$

and

$\tau \dot{z}_{\mathrm{demo}}=\alpha_{z}\left(\beta_{z}\left(y_{m}-y_{\mathrm{demo}}\right)-z_{\mathrm{demo}}\right)$,

the learning problem is formulated to find the parameters $\mathbf{w}_{k}$ in Eq. (6) using incremental locally weighted regression technique [30] in which $\mathbf{w}_{i}$ is updated by

$\mathbf{w}_{k}^{t+1}=\mathbf{w}_{k}^{t}+\Psi_{k} \mathbf{P}_{k}^{t+1} \tilde{\mathbf{v}} e_{k}$,

where

$\mathbf{P}_{k}^{t+1}=\frac{1}{\lambda}\left(\mathbf{P}_{k}^{t}-\frac{\mathbf{P}_{k}^{t} \tilde{\mathbf{v}} \tilde{\mathbf{v}}^{T} \mathbf{P}_{k}^{t}}{\left(\lambda / \Psi_{k}\right)+\tilde{\mathbf{v}}^{T} \mathbf{P}_{k}^{t} \tilde{\mathbf{v}}}\right)$,

$e_{k}=f_{\text {target }}-\mathbf{w}_{k}^{T} \tilde{\mathbf{v}}$ 
and $\lambda \in[0,1]$ is a forgetting factor. We chose this locally weighted regression framework as it can automatically find the correct number of necessary basis function, and can tune the $h_{k}$ parameters of each Gaussian kernel function (7) to achieve higher function approximation accuracy. Moreover, it learns the parameters $\mathbf{w}_{k}$ of every local model $k$ totally independent of all other local models, which minimizes interference between local models and provides a means to robustly classify a rhythmic pattern with the help of the parameters $\mathbf{w}_{k}$ [22].

\section{Rhythmic dynamical movement primitives as a CPG}

We use the rhythmic dynamical movement primitives introduced in Section 3.1 as a CPG for biped locomotion. Fig. 1 illustrates the proposed control architecture in this paper. Each joint is equipped with a movement primitive which generates the desired joint trajectory $\theta_{\text {des }}$. We define the index and the corresponding name of the joint as Left hip $(i=1$, L_HIP), and Left knee ( $i=2$, L_KNEE), Right hip $(i=3$, R_HIP), and Right knee ( $i=4$, R_KNEE). An additional oscillator $\left(\phi_{\text {ref }}\right)$ is allocated to provide a reference phase signal to the limb oscillators, which is adjusted by the ground contact information at the instance of heel strike. Section 4.1 introduces coupling to the oscillators of the movement primitives to achieve the desired phase relationship between the

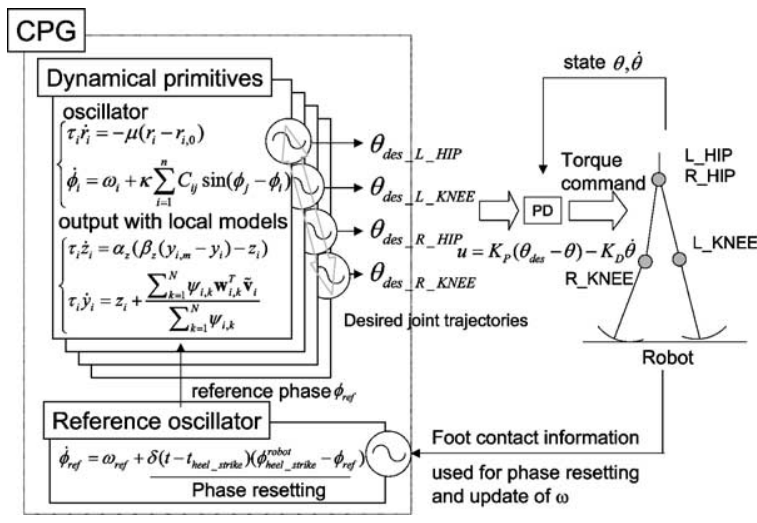

Fig. 1. Proposed control architecture for biped locomotion with dynamical movement primitives. limbs. Section 4.2 proposes a frequency adaptation algorithm of the learned periodic movements through the interaction among the coupled oscillators, robot and environment.

\subsection{Inter- and intra-limb phase coordination}

We introduce coupling among the oscillators to regulate the desired phase relationship between the limbs of the robot. This kind of coupling is motivated from a biological point of view where it has been hypothesized that coupling among neural oscillators plays an important role in coordinating the desired phase relationship of limb movements in locomotion and gait transition [31].

Consider the following coupling terms for the oscillator $i$ :

$\dot{\phi}_{i}=\omega_{i}+\kappa \sum_{i=1}^{N} C_{i j} \sin \left(\phi_{j}-\phi_{i}\right)$,

where $\kappa$ is a positive constant gain, and $C_{i j}$ is an element of the $n \times n$ matrix $\mathbf{C}$ which characterizes the coupling with other oscillators. This form of coupling appears in various studies of coupled oscillators and their application, e.g., [23-26,32,33]. In this paper, we employ the formulation in Ref. [23] to specify the desired phase relationship. In Ref. [23], $\mathbf{C}$ is defined to be a symmetric matrix where the diagonal elements are $C_{i i}=0$ for all $i$, and off-diagonal elements $C_{i j}$ are chosen as follows:

- $C_{i j}=1$ : oscillators $i$ and $j$ are designed to be in phase such that $\phi_{i}-\phi_{j}=0(\bmod 2 \pi)$.

- $C_{i j}=-1$ : oscillators $i$ and $j$ are designed to be out of phase such that $\phi_{i}-\phi_{j}=\pi(\bmod 2 \pi)$.

As noted in Ref. [23], an arbitrary phase difference other than 0 or $\pi$ can be specified by introducing a change of coordinates, or equivalent to having an offset in the coupling terms.

In this paper, we design the desired phase difference among the canonical oscillators such that the links of each leg move in phase (with zero phase difference), and the left and right legs move out of phase (with $\pi$ phase difference) by defining the phase of the oscillator as $\phi_{i}=0$ at the instance of heel strike of the corresponding leg. More specifically, we require $\phi_{1}-\phi_{2}=0, \phi_{3}-\phi_{4}=0, \phi_{1}-\phi_{3}=\pi$, and 
$\phi_{2}-\phi_{4}=\pi$. Thus, the connection matrix $\mathbf{C}$ is chosen to be

$\mathbf{C}=\left[\begin{array}{cccc}0 & 1 & -1 & -1 \\ 1 & 0 & -1 & -1 \\ -1 & -1 & 0 & 1 \\ -1 & -1 & 1 & 0\end{array}\right]$

\subsection{Frequency adaptation of locomotion}

Section 4.1 introduced internal coupling of the oscillators to coordinate the phase difference among the limbs of the robot. This section considers interaction between the environment and the CPG to achieve self-tuning of the natural frequency of the oscillators and synchronization of the CPG with the periodic behavior of the robot.

\subsubsection{Synchronization of coupled oscillators with frequency adaptation}

Before introducing the proposed frequency adaptation law for the biped robot CPG, let us consider the behavior of the following dynamics of two coupled oscillators:

$\dot{\phi}_{1}=\omega_{1}+K_{1}\left(\phi_{2}-\phi_{1}\right)$,

$\dot{\phi}_{2}=\omega_{2}+K_{2}\left(\phi_{1}-\phi_{2}\right)$,

where $\omega_{1}, \omega_{2}>0$ are natural frequencies of the oscillators, and $K_{1}, K_{2}$ are positive coupling constants. Then, the oscillators run with the phase difference $\psi^{*}=\phi_{2}-\phi_{1}=\left(\omega_{2}-\omega_{1}\right) /\left(K_{1}+K_{2}\right)$ at the coupled frequency $\omega^{*}=\left(K_{2} \omega_{1}+K_{1} \omega_{2}\right) /\left(K_{1}+K_{2}\right)$ when they are entrained. When $\omega_{1} \neq \omega_{2}$, the phase difference $\psi=\phi_{2}-\phi_{1}$ remains non-zero. However, if $\omega_{1}=\omega_{2}$, then the phase difference of these oscillators will be zero. Thus, we introduce an update law of the natural frequency $\omega_{1}$ to achieve synchronization of these oscillators with zero phase difference:

$\dot{\phi}_{1}(t)=\omega_{1}(t)+K_{1}\left(\phi_{2}(t)-\phi_{1}(t)\right)$,

$\dot{\omega}_{1}(t)=K\left(\omega_{2}-\omega_{1}(t)\right)$,

$\dot{\phi}_{2}(t)=\omega_{2}+K_{2}\left(\phi_{1}(t)-\phi_{2}(t)\right)$,

where $K$ is a positive constant. It is straightforward to see that $\omega_{1} \rightarrow \omega_{2}$ as $t \rightarrow \infty$. Thus, the phase difference will be zero such that $\psi=\phi_{2}-\phi_{1} \rightarrow 0$.

\subsubsection{Frequency update law and phase resetting of $C P G$}

In this section, we introduce an adaptation algorithm of the CPG in order to adjust the frequency of the learned periodic motions by the robot through the interaction among the CPG, robot and environment. As depicted in Fig. 1, the proposed control system can be regarded as a coupling of the CPG and the mechanical oscillator (robot) which is analogous to the coupled oscillator system discussed in Section 4.2.1.

For this purpose, we first introduce a reference oscillator $\left(\phi_{\text {ref }}\right)$ which will be synchronized with the locomotion of the robot through the adaptation mechanism described below. This reference oscillator can be considered as a phase estimator of locomotion by the discrete heel strike information detected by foot switches. Then, additional coupling is introduced to the limb oscillators with $\phi_{\text {ref }}$ to achieve the desired relative phase $\phi_{1}=\phi_{2}=\phi_{\text {ref }}$ and $\phi_{3}=\phi_{4}=\phi_{\text {ref }}+\pi$.

Motivated by the synchronization mechanism of the coupled oscillators in Section 4.2.1, we propose the following phase resetting and frequency update law. They can be interpreted as a discretized version of phase coupling (14) and frequency update (15) at the instance of heel strike:

$$
\begin{aligned}
& \dot{\phi}_{\text {ref }}=\hat{\omega}_{\text {ref }}^{n}+\delta\left(t-t_{\text {heel strike }}\right)\left(\phi_{\text {heel strike }}^{\text {robot }}-\phi_{\text {ref }}\right), \\
& \hat{\omega}_{\text {ref }}^{n+1}=\hat{\omega}_{\text {ref }}^{n}+K\left(\omega_{\text {measured }}^{n}-\hat{\omega}_{\text {ref }}^{n}\right),
\end{aligned}
$$

where $\delta$ is the Dirac's delta function, $n$ is the number of steps, and $\phi_{\text {heel strike }}^{\text {robot }}$ is the phase of the mechanical oscillator (robot) at heel strike defined as $\phi_{\text {heel strike }}^{\text {robot }}=$ 0 at the heel strike of the left leg, and $\phi_{\text {heel strike }}^{\text {robot }}=$ $\pi$ at the heel strike of the right leg. $\omega_{\text {measured }}^{n}$ is the measured frequency of locomotion defined by

$\omega_{\text {measured }}^{n}=\frac{\pi}{T_{\text {measured }}^{n}}$,

where $T_{\text {measured }}^{n}$ is the stepping period of locomotion (half period with respect to the oscillator). At the same time, natural frequencies of all the limb oscillators $\omega_{i}$ are updated at the instance of heel contact such that $\omega_{i}=\hat{\omega}_{\text {ref }}^{n+1}$. Note that it is possible to directly introduce phase resetting to the limb oscillators as seen in Ref. [26]. However, introduction of a reference oscillator allows phase estimation depending on multiple events and multi-modal information. Moreover, continuous phase coupling of the limb oscillators with the 

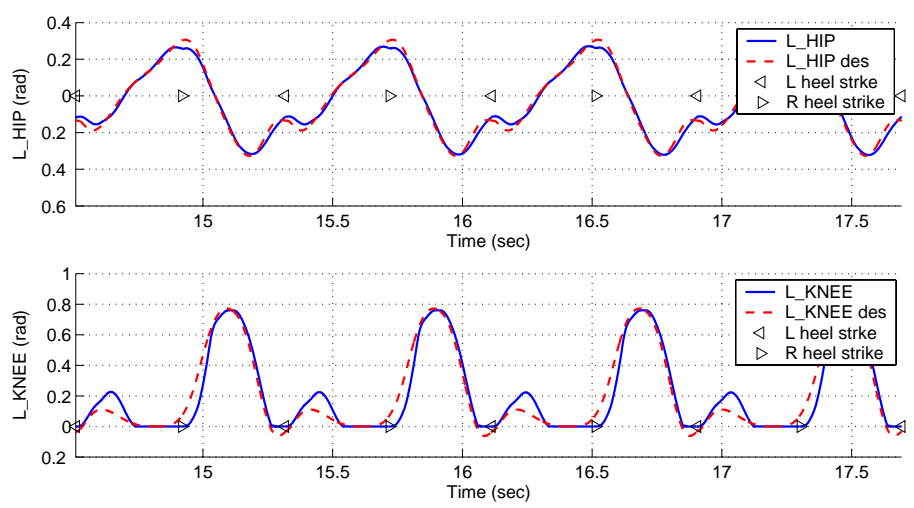

Fig. 2. Joint trajectories for the left leg and heel strike timing for four periods (eight steps) of walking (simulation).

reference oscillator having phase resetting alleviates the problem of discontinuity to the desired joint trajectories.

The phase resetting algorithm (17) is motivated from a biological perspective as well as a mathematical point of view. Phenomena of phase resetting or phase shift are observed in many biological oscillators resulting from external perturbations, e.g., circadian pacemakers, biochemical oscillators, and human finger tapping neural networks [27]. Phase resetting is related to the stability properties of neural rhythms, which can be analyzed by examining the phase-dependent responses against perturbations. A recent study [34] investigated the role of phase resetting in biped locomotion. Numerical studies in Ref. [34] suggest possible contribution of phase resetting during walking to gait stability against external perturbations.

\section{Numerical simulations}

As a demonstrated trajectory, we use the motion capture data of human walking in Ref. [35] (29-year-old male, $173 \mathrm{~cm}, 83.5 \mathrm{~kg}$, right hip and knee). We identified the period and frequency of this pattern by the power spectrum estimation with FFT and autocorrelation as $T=1.17 \mathrm{~s}$ and $f=$ $1 / T=0.855 \mathrm{~Hz}$, respectively. The dynamics of the robot are derived using SD/FAST ${ }^{1}$ and integrated using the Runge-Kutta algorithm at $1 \mathrm{~ms}$ step size.

\footnotetext{
${ }^{1}$ http://www.sdfast.com
}

The ground contact force is calculated using a linear spring-damper model. A low-gain PD controller is used at each joint to track the desired trajectory which is the output of the movement primitive.

A walking pattern from the demonstrated trajectory is learned with the dynamical primitives. We manually designed the desired trajectory for the initial step of locomotion from a standing position at rest, and the proposed CPG controller is activated at heel contact of the first step. The amplitude parameter of the dynamical primitives is set to $r_{0}=0.7$, and the offset $y_{m}=0.375$ is introduced to the knee joints. For the scaling of the natural frequency of the oscillator, the adaptation law proposed in Section 4.2.2 is used with the initial frequency of $\omega=4.83 \mathrm{rad} / \mathrm{s}$ (period of oscillation is $1.3 \mathrm{~s}$ ). These parameters are determined empirically from trial and error.

Fig. 2 illustrates the desired and actual joint trajectories for the left leg, and the timing of heel strike after a stable pattern was learned by the phase resetting algorithm. Fig. 3 shows the torque command for the left leg, which indicates that the knee joint swings passively since it requires almost no torque (see $t=$ 15.1-15.3 s). Fig. 4 depicts one step of walking. Fig. 5 (left) shows the adaptation of the period of locomotion and Fig. 5 (right) shows the learning curve of the frequency of the CPG with different coupling constants $K=0.2,0.5$ and 0.8 in Eq. (18). The stepping period approached $0.387 \mathrm{~s}$, and the resultant CPG frequency was $\omega=8.12 \mathrm{rad} / \mathrm{s}$, which roughly corresponds to the natural frequency of the swing leg modeled as a simplified linear pendulum, using the proposed adaptation law. 

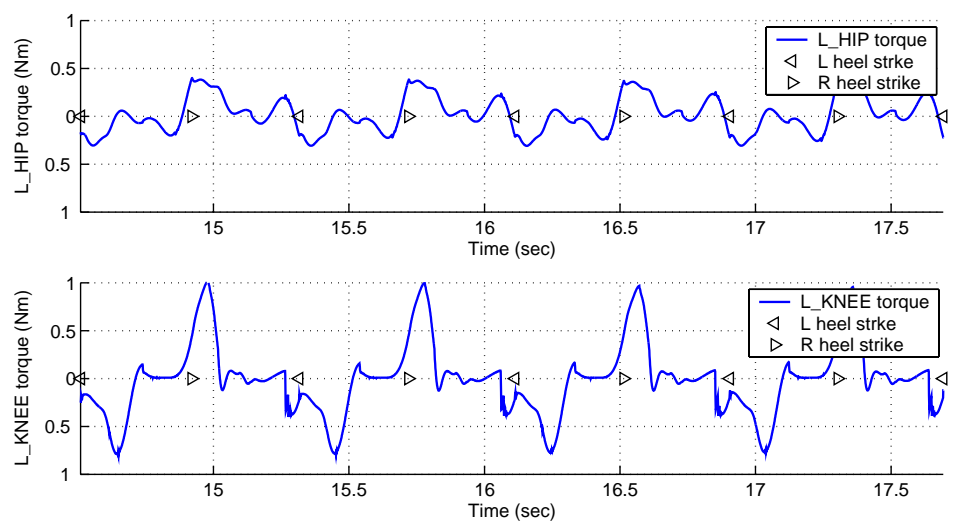

Fig. 3. Torque command to the left hip and knee joints for four periods (eight steps) of walking (simulation).
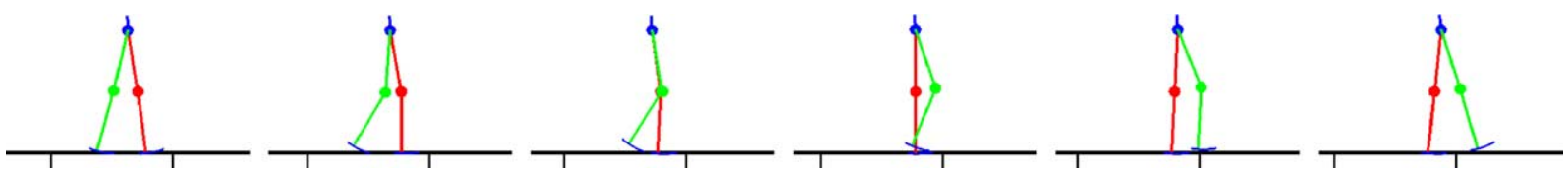

Fig. 4. Snapshots of walking simulation for one step at 15 frames/s ( 1 frame $\approx 66 \mathrm{~ms})$.

Robustness against external perturbations is evaluated by pushing the robot forward and backward with external forces. Forces are applied for a duration of $0.1 \mathrm{~s}$ at different timing during a single step (at an interval of $0.1 \mathrm{rad}$ from 0 to $2 \pi$ of the phase of the reference oscillator). When a forward perturbing force is applied, the robot could cope with up to $9.1 \mathrm{~N}(\max )$ at $\phi=1.1 \mathrm{rad}$ and $2.2 \mathrm{~N}(\mathrm{~min})$ at $\phi=2.7 \mathrm{rad}$ of the perturbing forces. When a backward perturbing force is applied, the robot could cope with up to $-2.4 \mathrm{~N}(\max )$

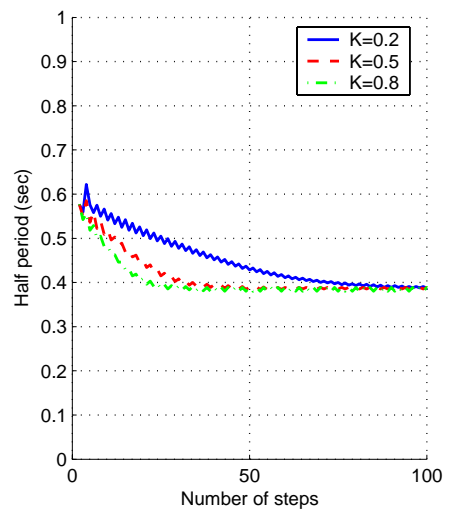

at $\phi=4.9 \mathrm{rad}$ and $-1.0 \mathrm{~N}(\mathrm{~min})$ at $\phi=0.4$ and $\phi=$ $0.5 \mathrm{rad}$ of the perturbing forces. In contrast, without phase resetting, the robot only could cope with much smaller disturbances, for example, the robot only tolerated up to $3.9 \mathrm{~N}$ of the forward perturbing force applied at $\phi=1.1$.

The simulation results demonstrate self-adaptation of the frequency of locomotion and robustness of walking against disturbance by the proposed algorithm.

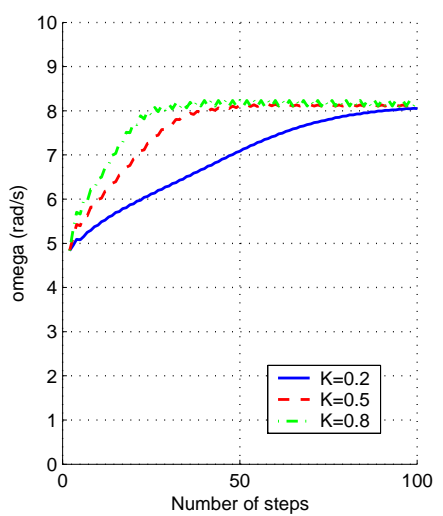

Fig. 5. Frequency adaptation of walking via entrainment (simulation). Left: adaptation of period. Right: learning curve of the frequency of the CPG. 

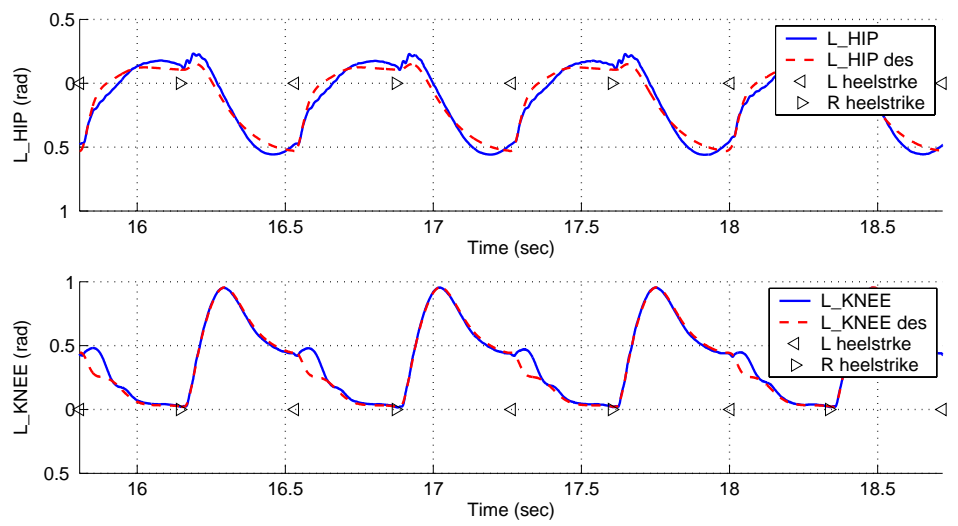

Fig. 6. Joint trajectories for the left leg and heel strike timing for four periods (eight steps) of walking (experiment).

\section{Experimental implementation}

We implemented the proposed control framework on our biped robot. In the experimental implementation, our initial attempt to achieve biped locomotion using the human demonstrated trajectory was not successful largely due to mechanical limitation of the experimental system and discrepancy in the ground contact condition between simulations and experiments. Thus, we used another target trajectory which was experimentally obtained from an actual trajectory of successful robot locomotion using a state machine controller. The state machine controller is designed to coordinate the leg movements with the physical state of the legged system based on the idea presented in Ref. [36].
To initiate locomotion in the experiments, we first suspended the robot with the legs swinging in the air, and then placed the robot on the ground manually. Thus, the initial condition of each run was not consistent, and occasionally the robot could not start walking or fell over after a couple of steps when the timing was not appropriate. Fig. 6 illustrates the desired and actual joint trajectories for the left leg, and the timing of heel strike. Fig. 7 shows the torque command for the left leg. Some oscillation in the torque command for the hip joint can be seen. This is due to noisy joint velocity signals obtained from numerically differentiated joint angles measured by optical encoders. We are currently planning to use gyros to obtain smoother joint velocities to improve the performance of the tracking controller. Note that a limit on the torque command
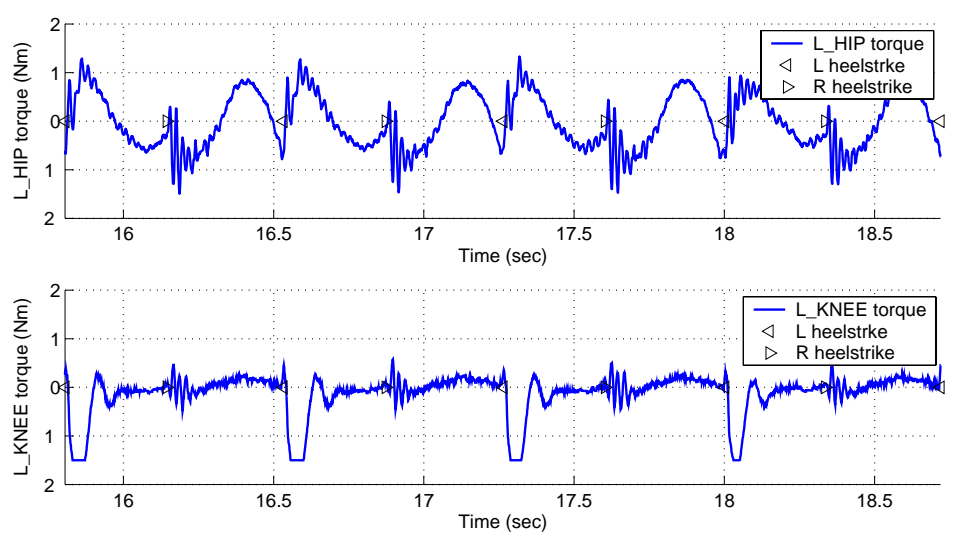

Fig. 7. Torque command to the left hip and knee joints for four periods (eight steps) of walking (experiment). 

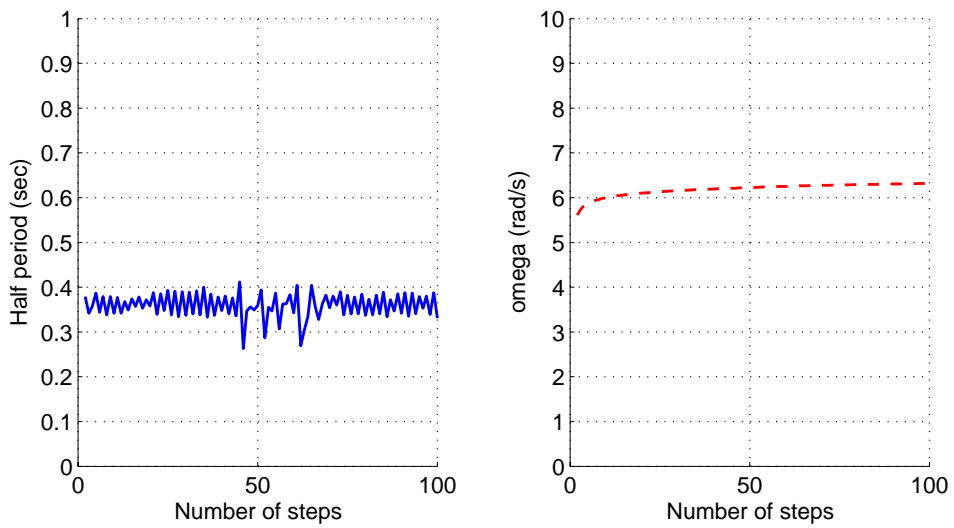

Fig. 8. Frequency adaptation of walking via entrainment (experiment). Left: adaptation of period. Right: learning curve of the frequency of the $\mathrm{CPG}$

is imposed at $\pm 1.5 \mathrm{Nm}$. Fig. 8 (left) shows the period adaptation and Fig. 8 (right) shows the learning curve of the frequency of the CPG. Stepping period for a typical walking experiment was around $0.37 \mathrm{~s}$. In this experiment, the initial frequency of the oscillator was set to $\omega=5.71 \mathrm{rad} / \mathrm{s}$ (period of oscillation is $1.1 \mathrm{~s}$ ), and the adaptation gain in Eq. (18) was decreased according to an annealing procedure $K=K_{0} / n$, where $K_{0}=0.05$ and $n$ is the number of steps, as is needed in most gradient descent procedure. We introduced an offset $\alpha$ for phase resetting

$\dot{\phi}_{\text {ref }}=\hat{\omega}^{n}+\delta\left(t-t_{\text {heel strike }}\right)\left(\phi_{\text {heel strike }}^{\text {robot }}-\phi_{\text {ref }}+\alpha\right)$

to adjust the timing of foot contact, where $\alpha$ is chosen to be $\alpha=0.8 \mathrm{rad}$. These parameters are determined empirically. Note that phase resetting with an offset effectively changes the period of oscillation.
Robustness of the proposed algorithm is evaluated by testing walking over surfaces with different friction properties such as carpet, cork sheet (3 $\mathrm{mm}$ thick) and a seesaw-like metal plate ( $2 \mathrm{~mm}$ thick). The metal plate was placed so that the inclination of the slope slightly changes like a seesaw when the robot walks over it (the height of the center is $7 \mathrm{~mm}$ ). The robot could deal with the change in the environment as depicted in Fig. 9.

Note that even if we use the learned trajectory from the actual robot walking pattern, the robot could not walk by just replaying it as a desired trajectory. Phase resetting using foot contact information was necessary. This implies that appropriate on-line adjustment of the phase of the CPG by sensory feedback from the environment is essential to achieve successful locomotion. In addition, empirically we found that the proposed controller achieved much more robust walking compared to the state machine-based controller which we originally designed.
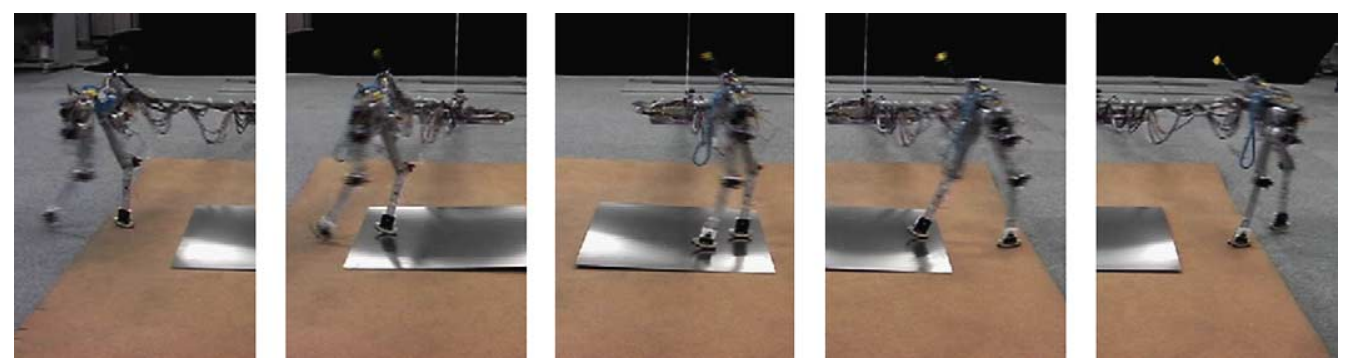

Fig. 9. Walking over surfaces with different friction properties and a seesaw-like metal sheet with a slight change in the slope. 


\section{Conclusion}

In this paper, we proposed a method for learning biped locomotion from demonstration and its frequency adaptation using dynamical movement primitives. In the dynamical movement primitives, kinematic movement plans are described in a set of non-linear differential equations with well-defined attractor dynamics, and demonstrated trajectories are learned using locally weighted regression. Specifically, we use rhythmic dynamical movement primitives based on coupled phase oscillators as a CPG, and introduced a frequency adaptation algorithm through interactions among the CPG, mechanical system and the environment motivated by the synchronization of coupled oscillators. Numerical simulations and experimental result demonstrate the effectiveness of the proposed control algorithm to achieve steady-state walking roughly at the natural frequency of the coupled system. We also evaluated robustness against disturbance in numerical simulations and experiments.

Future work will address initiation and termination of walking, and on-line balance compensation. We will also consider collection of human's walking data under various behavioral conditions. In our current study, we used a simple phase resetting mechanism in which the phase of the CPG is forced to be reset to a specific value at the instance of heel strike regardless of the current phase of the CPG. In the future, we are interested in the generalization of the idea of phase resetting to determine phase-dependent reaction against external perturbations such as recovery from stumbling by designing an appropriate phase resetting curve [27]. Formal mathematical analysis will be required to understand the principle of periodic stability of a limit cycle solution to the dynamics of a combined oscillator and mechanical system. In the long run, we are hopeful that our approach may provide insight into a theoretically sound design principle of biped locomotion control to achieve human-like natural walking.

\section{Acknowledgements}

We would like to thank Auke Ijspeert at EPFL, Swiss Federal Institute of Technology, Lausanne and Seiichi Miyakoshi of the Digital Human Research Center, AIST, Japan, and Chris Atkeson at Carnegie
Mellon University, for valuable discussions and helpful comments.

This research was supported in part by National Science Foundation grants ECS-0325383, IIS-0312802, IIS-0082995, ECS-0326095, ANI-0224419, a NASA grant AC\#98-516, an AFOSR grant on Intelligent Control, the Communications Research Laboratory of Japan, the ERATO Kawato Dynamic Brain Project, funded by the Japan Science and Technology Agency, and the ATR Computational Neuroscience Laboratories.

\section{References}

[1] M. Vukobratović, B. Borovac, D. Surla, D. Stokić, Biped Locomotion-Dynamics, Stability, Control and Application, Springer, 1990.

[2] S. Kagami, T. Kitagawa, K. Nishiwaki, T. Sugihara, M. Inaba, $\mathrm{H}$. Inoue, A fast dynamically equilibrated walking trajectory generation method of humanoid robot, Autonomous Robots 12 (2002) 71-82.

[3] A. Takanishi, M. Tochizawa, H. Karaki, I. Kato, Dynamic biped walking stabilized with optimal trunk and waist motion, in: Proceedings of the IEEE/RSJ International Workshop on Intelligent Robots and Systems, 1989, pp. 561-566.

[4] K. Hirai, M. Hirose, Y. Haikawa, T. Takenaka, The development of honda humanoid robot, in: Proceedings of the IEEE International Conference on Robotics and Automation, 1998, pp. 1321-1326.

[5] S. Kagami, F. Kanehiro, Y. Tamiya, M. Inaba, H. Inoue, Autobalancer: an online dynamic balance compensation scheme for humanoid robots, in: B.R. Donald, K. Lynch, D. Rus (Eds.), Algorithmic and Computational Robotics: New Directions, A K Peters Ltd., 2001, pp. 329-340.

[6] J. Yamaguchi, A. Takanishi, I. Kato, Development of a biped walking robot compensating for three-axis moment by trunk motion, in: Proceedings of the IEEE/RSJ International Conference on Intelligent Robots and Systems, 1993, pp. 187-192.

[7] K. Yoneda, S. Hirose, Tumble stability criterion of integrated locomotion and manipulation, in: Proceedings of the IEEE/RSJ International Conference on Intelligent Robots and Systems, 1996, pp. 870-876.

[8] S. Mochon, T.A. McMahon, Ballistic walking, Journal of Biomechanics 13 (1980) 49-57.

[9] K. Matsuoka, Sustained oscillations generated by mutually inhibiting neurons with adaptation, Biological Cybernetics 52 (1985) 367-376.

[10] G. Taga, Y. Yamaguchi, H. Shimizu, Self-organized control of bipedal locomotion by neural oscillators in unpredictable environment, Biological Cybernetics 65 (1991) 147-159.

[11] S. Miyakoshi, G. Taga, Y. Kuniyoshi, A. Nagakubo, Three dimensional bipedal stepping motion using neural oscillators-towards humanoid motion in the real world, in: 
Proceedings of the IEEE/RSJ International Conference on Intelligent Robots and Systems, 1998, pp. 84-89.

[12] K. Hase, N. Yamazaki, Computational evolution of human bipedal walking by a neuro-musculo-skeletal model, Artificial Life and Robotics 3 (1999) 133-138.

[13] G. Endo, J. Morimoto, J. Nakanishi, G. Cheng, An empirical exploration of a neural oscillator for biped locomotion, in: Proceedings of the IEEE International Conference on Robotics and Automation, 2004, pp. 3036-3042.

[14] Y. Fukuoka, H. Kimura, A.H. Cohen, Adaptive dynamic walking of a quadruped robot on irregular terrain based on biological concepts, International Journal of Robotics Research 22 (3-4) (2003) 187-202.

[15] S. Miyakoshi, M. Yamakita, K. Furuta, Juggling control using neural oscillators, in: Proceedings of the IEEE/RSJ International Conference on Intelligent Robots and Systems, 1994, pp. 1186-1193.

[16] S. Kotosaka, S. Schaal, Synchronized robot drumming by neural oscillator, in: Proceedings of the International Symposium on Adaptive Motion of Animals and Machines, 2000.

[17] M.M. Williamson, Neural control of rhythmic arm movements, Neural Networks 11 (1998) 1379-1394.

[18] J. Pratt, G. Pratt, Intuitive control of a planar bipedal walking robot, in: Proceedings of the IEEE International Conference on Robotics and Automation, 1998, pp. 2014-2021.

[19] H. Miyamoto, S. Schaal, F. Gandolfo, Y. Koike, R. Osu, E. Nakano, Y. Wada, M. Kawato, A kendama learning robot based on bi-directional theory, Neural Networks 9 (1996) 1281-1302.

[20] H. Miyamoto, M. Kawato, A tennis serve and upswing learning robot based on bi-directional theory, Neural Networks 11 (1998) 1317-1329.

[21] S. Schaal, Is imitation learning the route to humanoid robots? Trends in Cognitive Sciences 3 (6) (1999) 233-242.

[22] A. Ijspeert, J. Nakanishi, S. Schaal, Learning attractor landscapes for learning motor primitives, in: S. Becker, S. Thrun, K. Obermayer (Eds.), Advances in Neural Information Processing Systems, vol. 15, MIT-Press, 2003, pp. 1547-1554.

[23] E. Klavins, D.E. Koditschek, Phase regulation of decentralized cyclic robotic systems, International Journal of Robotics Research 21 (3) (2002) 257-275.

[24] S. Ito, H. Yuasa, Z. wei Luo, M. Ito, D. Yanagihara, A mathematical model of adaptation in rhythmic motion to environmental changes, in: Proceedings of the IEEE International Conference on Systems, Man and Cybernetics, 1997, pp. 275-280.

[25] K. Tsujita, K. Tsuchiya, A. Onat, Adaptive gait pattern control of a quadruped locomotion robot, in: Proceedings of the IEEE/RSJ International Conference on Intelligent Robots and Systems, 2001, pp. 2318-2325.

[26] K. Tsuchiya, S. Aoi, K. Tsujita, Locomotion control of a biped locomotion robot using nonlinear oscillators, in: Proceedings of the IEEE/RSJ International Conference on Intelligent Robots and Systems, 2003, pp. 1745-1750.
[27] M. Kawato, Transient and steady state phase response curves of limit cycle oscillators, Journal of Mathematical Biology 12 (1981) 13-30.

[28] J. Peters, S. Vijayakumar, S. Schaal, Reinforcement learning for humanoid robotics, in: Proceedings of the Third IEEE-RAS International Conference on Humanoid Robots, 2003.

[29] J. Morimoto, G. Zeglin, C.G. Atkeson, Minimax differential dynamic programming: application to a biped walking robot, in: Proceedings of the IEEE/RSJ International Conference on Intelligent Robots and Systems, 2003, pp. 1927-1932.

[30] S. Schaal, C.G. Atkeson, Constructive incremental learning from only local information, Neural Computation 10 (8) (1998) 2047-2084.

[31] A.J. Ijspeert, A connectionist central pattern generator for the aquatic and terrestrial gaits of a simulated salamander, Biological Cybernetics 84 (5) (2001) 331-348.

[32] Y. Kuramoto, Chemical Oscillations, Waves, and Turbulence, Springer-Verlag, 1984.

[33] K. Sekiyama, J. Nakanishi, I. Takagawa, T. Higashi, T. Fukuda, Self-organizing control of urban traffic signal network, in: Proceedings of the IEEE International Conference on Systems, Man and Cybernetics, 2001, pp. 2481-2486.

[34] T. Yamasaki, T. Nomura, S. Sato, Possible functional roles of phase resetting during walking, Biological Cybernetics 88 (6) (2003) 468-496.

[35] Y. Ehara, S. Yamamoto, Introduction to Body-DynamicsAnalysis of Gait and Gait Initiation, Ishiyaku Publishers, 2002 (in Japanese).

[36] J.K. Hodgins, Biped gait transitions, in: Proceedings of the IEEE International Conference on Robotics and Automation, 1991, pp. 2092-2097.

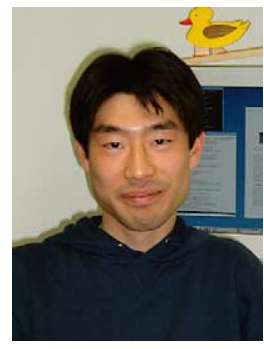

Jun Nakanishi received the B.E. and M.E. degrees both in mechanical engineering from Nagoya University, Nagoya, Japan, in 1995 and 1997, respectively. He received the Ph.D. degree in engineering from Nagoya University in 2000 . He also studied in the Department of Electrical Engineering and Computer Science at the University of Michigan, Ann Arbor, USA, from 1995 to 1996 . He was a Research Associate at the Department of Micro System Engineering, Nagoya University, from 2000 to 2001, and was a presidential postdoctoral fellow at the Computer Science Department, the University of Southern California, Los Angeles, USA, from 2001 to 2002. He joined ATR Human Information Science Laboratories, Kyoto, Japan, in 2002. He is currently a researcher at ATR Computational Neuroscience Laboratories and with the Computational Brain Project, ICORP, Japan Science and Technology Agency. His research interests include motor learning and control in robotic systems. He received the IEEE ICRA 2002 Best Paper Award. 


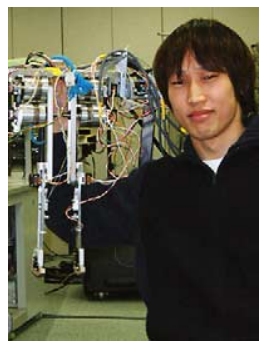

Jun Morimoto received his B.E. in computer-controlled mechanical systems from Osaka University, Osaka, Japan, in 1996, M.E. in information science from Nara Institute of Science and Technology, Nara, Japan, in 1998, and Ph.D. in information science from Nara Institute of Science and Technology, Nara, Japan, in 2001. He was a Research Assistant at Kawato Dynamic Brain Project, ERATO, JST, from 1999 to 2001. He was a postdoctoral fellow at the Robotics Institute, Carnegie Mellon University, Pittsburgh, PA, from 2001 to 2002. He is currently a researcher at ATR Computational Neuroscience Laboratories, Kyoto, Japan, and with the Computational Brain Project, ICORP, Japan Science and Technology Agency. He is a member of Japanese Neural Network Society, and Robotics Society of Japan. His research interests include reinforcement learning and robotics.

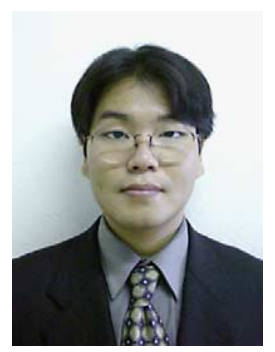

Gen Endo received his B.E. and M.E. degrees both in Mechano-Aerospace Engineering from Tokyo Institute of Technology, Tokyo, Japan, in 1996 and 1998, respectively. He received the Ph.D. degree in engineering from Tokyo Institute of Technology in 2000. He joined Sony Corporation, Tokyo, Japan, in 2000. He is currently a collaborative researcher at ATR Computational Neuroscience Laboratories, Kyoto, Japan. He is a member of Robotics Society of Japan. He received the Best Paper Award of Robotics Society of Japan in 2002. His research interests include biologically inspired mobile robot, mechanical design and control.

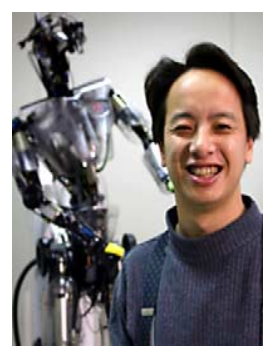

Gordon Cheng is the head of the Department of Humanoid Robotics and Computational Neuroscience, ATR Computational Neuroscience Laboratories, Kyoto, Japan, and with the Computational Brain Project, ICORP, Japan Science and Technology Agency. Before taking up this position, he held fellowships from the Center of Excellence (COE), Science, and Technology Agency (STA) of Japan. Both of these fellowships were taken at the Humanoid Interaction Laboratory, Intelligent Systems Division at the ElectroTechnical Laboratory (ETL), Japan. At ETL he played a major role in developing a completely integrated humanoid robotics system. He received a Ph.D. in systems engineering from the Department of Systems Engineering, The Australian National University, and Bachelor and Master degrees in Computer Science from the University of Wollongong, Australia. His industrial experience includes consultancy to and as a national systems manager for a major transport company. He was also the director of the company, G.T.I. Computing, specializing in networking/transport management systems in Australia. His research interests include humanoid robotics, biomimetic of human vision, computational neuroscience of vision, action understanding, human-robot interaction, active vision, mobile robot navigation and object-oriented software construction. $\mathrm{He}$ is a society member of the IEEE Robotics \& Automation and Computer Society. He is on the editorial board of the International Journal of Humanoid Robotics. He is a program co-chair for the next IEEE International Conference on Humanoid Robots.

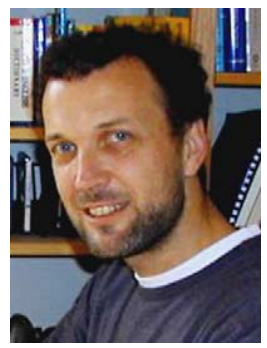

Stefan Schaal is an Associate Professor at the Department of Computer Science and the Neuroscience Program at the University of Southern California, and an Invited Researcher at the ATR Computational Neuroscience Laboratory in Japan, where he held an appointment as Head of the Computational Learning Group during an international ERATO project, the Kawato Dynamic Brain Project (ERATO/JST). He is also an Adjunct Assistant Professor at the Department of Kinesiology of the Pennsylvania State University. Before joining USC, Dr. Schaal was a postdoctoral fellow at the Department of Brain and Cognitive Sciences and the Artificial Intelligence Laboratory at MIT, an Invited Researcher at the ATR Human Information Processing Research Laboratories in Japan, and an Adjunct Assistant Professor at the Georgia Institute of Technology. Dr. Schaal's research interests include topics of statistical and machine learning, neural networks, computational neuroscience, non-linear dynamics, non-linear control theory, and biomimetic robotics. He applies his research to problems of artificial and biological motor control and motor learning, focusing on both theoretical investigations and experiments with human subjects and anthropomorphic robot equipment.

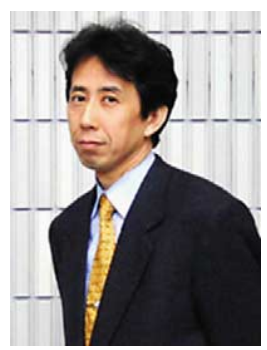

Mitsuo Kawato received the B.S. degree in physics from Tokyo University in 1976, the M.E. and Ph.D. degrees in biophysical engineering from Osaka University in 1978 and 1981, respectively. From 1981 to 1988 he was a faculty member and lecturer of Osaka University. From 1992 he became a department head of Department 3, ATR Human Information Processing Research Laboratories. From 2003, Director of ATR Computational Neuroscience Laboratories. From 2004, he has been jointly appointed as the Director of the Computational Brain Project, ICORP, JST. From 1996 to 2001 he was jointly appointed as a director of Kawato Dynamic Brain Project, ERATO, JST. He has been jointly appointed as visiting professor of Kanazawa Institute of Technology, Nara Institute of Science and Technology and Osaka University. For the last 15 
years he has been working in computational neuroscience. He was awarded Yonezawa founder's medal memorial special award of the Institute of Electronics, Information and Communication Engineers in 1991, outstanding research award of the International Neural Network Society in 1992, Osaka Science Prize in 1993, 10th Tsukahara Naka-akira Memorial Award in 1996 and Tokizane Toshihiko memorial award. He is a governing board member of Japanese Society of Neuroscience and Japan Neural Network Society and Member of Executive Committee of International Association for the Study of Attention and Performance. 